Volume 6

2020

\title{
Decoy Effects Improve Diversity Hiring
}

\author{
Nathan R. Kuncel \\ University of Minnesota - Twin Cities \\ Jeffrey A. Dahlke \\ Human Resources Research Organization
}

Follow this and additional works at: https://scholarworks.bgsu.edu/pad

Part of the Human Resources Management Commons, Industrial and Organizational Psychology Commons, and the Other Psychology Commons

How does access to this work benefit you? Let us know!

\section{Recommended Citation}

Kuncel, Nathan R. and Dahlke, Jeffrey A. (2020) "Decoy Effects Improve Diversity Hiring," Personnel Assessment and Decisions: Number 6 : Iss. 2 , Article 5.

DOI: https://doi.org/10.25035/pad.2020.02.005

Available at: https://scholarworks.bgsu.edu/pad/vol6/iss2/5

This Main Article is brought to you for free and open access by the Journals at ScholarWorks@BGSU. It has been accepted for inclusion in Personnel Assessment and Decisions by an authorized editor of ScholarWorks@BGSU. 


\title{
Decoy EfFects Improve Diversity HIRING
}

\author{
Nathan R. Kuncel ${ }^{1}$ and Jeffrey A. Dahlke ${ }^{2}$ \\ 1. University of Minnesota \\ 2. Human Resources Research Organization
}

ABSTRACT

\begin{abstract}
A growing literature demonstrates that when making choices among multiple options, decision makers are strongly influenced by the mere presence of additional options, even when those options are largely undesirable and are never actually selected. The effects of irrelevant options on decisions, often called decoy effects, have been observed in hiring and admissions decisions where the nature of a third candidate can radically shift preferences. In this study, we examine the influence of decoy effects on diversity hiring and extend research by examining choices with more than two organizational goals. Results indicate that the presence of a second candidate who meets diversity goals markedly increases how frequently decision makers indicate that they would make an offer to diverse candidates. This effect occurs even when decision makers must sacrifice some credential quality to obtain the diverse candidate. Overall, diverse candidates are more likely to receive offers when more than one diversity candidate is included in the finalist pool. The practical implications are clear: When a major organizational goal is to increase diversity, a policy that includes evaluating multiple diverse candidates in a final applicant slate should be considered.
\end{abstract}

judgment and decision making, personnel selection, diversity

$\square$

During a hiring or admissions process, decision makers are confronted with making choices among multiple people based on one or more pieces of information (e.g., credentials, skills, scores). In most cases, this involves juggling multiple goals, including considering the relative strengths of different applicants as well as acting in the interest of different organizational goals. Diversity is often considered one major organizational goal in hiring, with $86 \%$ of surveyed global corporations viewing it as at least a moderately important goal (SHRM, 2009). A number of strategies have been adopted to address diversity goals, including community outreach, retention-focused interventions, training, and targeted recruiting. Ultimately, however, organizational diversity often comes down to a decision to actually extend an offer to one applicant over another. It is here, at the final job offer decision, that we focus our attention.

When making a choice among multiple options, a large body of research demonstrates that decisions are heavily influenced by comparisons among options and that the features of these comparisons matter above and beyond the features of the options themselves (Huber \& Puto, 1983). Early work identified the phenomenon of preference reversals where decision makers reliably reversed their preferred choice depending on whether the options were evaluated separately or were paired and presented at the same time. Other work has considered how people make a final choice between two strong choices. This stream of research has demonstrated that the features of a third, but overall weak er, choice (known as a decoy) can influence which of the first two options is actually selected. In other words, these decoy effects occur when the third alternative strongly influences the choice between the first two but is never itself selected.

This same phenomenon has also been demonstrated when making hiring decisions (Highhouse, 1996). In most studies, two candidates are presented who differ on qualifications but are effectively tied overall. Included is a third candidate who represents the experimental manipulation. The third candidate (i.e., the decoy) is given credentials that are overall worse than either primary candidate, yet the decoy slightly exceeds one of the credentials for one of the primary candidates in each condition. This manipulation results in a strong shift in preference for one or the other candidate. The basic design used in Highhouse (1996) is presented in Table 1 . Note that the top two candidates remain effectively tied in each condition, but the preference choice is heavily influence by the properties of the third candidate, even though the third candidate is never selected and is clearly weaker than either of the top two choices.

\footnotetext{
Corresponding author:

Nathan R. Kuncel

75 East River Road

University of Minnesota

Minneapolis, MN 55455

Email: kunce001@umn.edu
} 
TABLE 1.

Decoy Effect Design From Highhouse (1996)

\begin{tabular}{lcc}
\hline Candidate & $\begin{array}{c}\text { Interview } \\
\text { rating }\end{array}$ & $\begin{array}{c}\text { Promotability } \\
\text { rating }\end{array}$ \\
\hline K. Martin & 5 & 80 \\
R. Davis & 7 & 57 \\
T. Doyle (Decoy K. Martin) & 4 & 80 \\
T. Doyle (Decoy R. Davis) & 7 & 46 \\
\hline
\end{tabular}

Comparisons are key in making decisions and this effect has replicated for both individual and group decision making (Slaughter et al., 2006) and in high fidelity hiring studies where attributes were inferred from nonquantitative information (Slaughter et al., 1999).

A number of explanations have been offered for these effects. Early explanations argued for a perceptual bias where adding an irrelevant option that increases the range of a dimension creates a bias that decreases the salience of that dimension. It was argued that this occurs because the perceived relative magnitude of the difference between the target and the competitor will be diminished, although the absolute difference is unchanged. This perceptual shift reduces a decision maker's focus on the range-extended dimension, which alters preferences. However, the magnitude of range extension associated with decoys does not appear to be important: Huber et al. (1982) and Huber and Puto (1983) both reported that extreme range decoys and less extreme decoys had had similar effects on preferences. Thus, range decoys seem to create ordinal shifts in perceived attractiveness within a dimension rather than scale-dependent shifts.

Loss aversion has been proposed as an explanation for attraction effects (Tversky \& Simonson, 1993). This explanation is based on the well-replicated finding that losses loom larger than gains (Kahneman \& Tversky, 1979). Decoys may function as reference points that decision makers use to calibrate their evaluations of other options (Highhouse, 1996; Tversky \& Kahneman, 1991). The presence of alternatives creates a comparison where the targeted alternative represents a gain on one characteristic and no loss on a second, whereas choosing the second alternative represents a larger gain on one characteristic but a loss on a second when compared to the decoy. In this study, we find that the presence of a decoy alters behavior, but the diversity choice still represents a loss on at least one dimension. This presents a different perspective on loss aversion as will be discussed later.

The ease-of-justification explanation (Park \& Kim, 2005; Simonson, 1989; Wedell \& Pettibone, 1996) proposes that attraction effects occur because the presence of an additional option (the decoy) provides decision makers with a reference point around which to craft a defensible argument for their choice. When a decoy is present, a decision maker can justify his or her choice of the target option on the basis of the target being demonstrably superior to the decoy, even though the target is not clearly superior to the competitor. It is worth noting that ease-of-justification explanations are not incompatible with loss-aversion explanations. The addition of another candidate may create an emergent value (Pettibone \& Wedell, 2000), allowing the decision maker to justify their decision (Simonson, 1989). For example, in the presence of a second diversity candidate, the primary diversity candidate may shift from being the "diversity candidate" to the "best diversity candidate." The decision to hire that candidate is then justified and defensible because the candidate is now the best in class.

Previous research has only focused on making choices among candidates to address a single organization goal: candidate strength. We believe that attraction effects may influence more realistic scenarios where multiple organizational goals are in play, including trying to hire a diverse work group. We are not alone in this idea: Highhouse (1997) speculated that the decoy effect would likely apply to hiring decisions with three faculty candidates, where the presence of two women and one man versus one woman and two men might result in an effect favoring the more strongly represented sex. Decision makers might make the choice for the "best man" or "best woman," depending on the context.

The importance of comparisons was highlighted by Bohnet et al. (2016), who demonstrated that joint comparisons of job candidates caused decision makers to more carefully evaluate the actual credentials of the applicant whereas separate evaluations tended to be associated with use of stereotypes. The consequence was fairer evaluations of applicants even when they didn't fit the gender stereotyped job. Although some jobs are always based on joint evaluations involving slates of candidates (like the faculty hires discussed by Highhouse), this study illustrates the potential importance of simple nudges in hiring (Sunstein \& Thaler, 2008).

This study differs from previous research in important ways. First, we are adding an additional dimension for consideration: applicant racial and ethnic diversity. Decision makers are given the paired goals of making good hires and increasing the racial and ethnic diversity of their team over time.

Second, we expand research in this area in two additional ways by considering more than three choice options across more than two dimensions per option. As a result, we do not use the traditional three applicant scenario seen in other studies. We contrast a baseline three applicant scenario in which none of the candidates dominate any of the others with a four candidate scenario where the added candidate is dominated by the baseline diverse candidate. 
This change is done to accommodate the additional goal of considering diversity. Only when we add the additional diverse candidate is one candidate able to dominate the others. In these conditions, the primary diversity candidate has both stronger credentials AND meets the diversity goal. We are aware of only two studies that have done either of these things (Ariely \& Wallsten, 1995; Huber \& Puto, 1983), and neither examined hiring decisions, let alone diversity.

Based on previous research and theory, we expect that the presence of an additional diversity candidate will increase the frequency of offers made to diverse candidates. At the same time, we expect that the credentials of the primary diversity candidate will also influence the frequency of offers, such that stronger candidates will receive more offers.

\section{METHOD}

This study is a $2 \times 2$ between-subjects design with the decision to hire or not hire the primary diversity candidate as the dependent variable. The first factor was the credential level of the primary diversity candidate relative to the other two candidates. This was varied at lower and higher credential levels. The second factor was the presence or absence of a second diversity candidate whose credentials were set to be lower than any of the candidates (conditions are displayed in Appendix A). In principle, the presence of the additional candidate (an additional option) should not increase the decision to hire the first diversity candidate (the principle of regularity; Rieskamp et al., 2006). In fact, the added candidate should be largely ignored in any decisions because the added candidate's credentials are not competitive with any of the primary candidates' credentials.

Research participants were told that their supervisor wanted them to consider both the credentials of the applicants but also have the goal of considering diversity in hiring. Specifically, they were told "Your organization is attempting to hire a new project manager. You have been given the goal of making a good hire. Your boss wants you to: increase the racial and ethnic diversity of your team over time [and] hire a candidate who knows the business and has good organizational skills." Each candidate was assigned ratings on the "know the business" and "organizational skill" credentials, and it was also indicated to participants whether each candidate was considered a "diversity candidate."

Previous research on decoy effects have yielded very large effects. However, in this study, with competing goals, we expected effects to be sizable but more modest in magnitude. Using the G*Power power-analysis program (Faul et al., 2009), we determined that a sample size of 217 would provide $80 \%$ power to detect an odds ratio of 2.0 (a medium-sized effect) in logistic regression. Given that our deci- sion-making scenarios included more candidates and more dimensions than previous personnel-selection decoy studies and that we were interested in testing two main effects, we aimed to recruit a final total sample of 300 participants for a more powerful analysis.

Research participants were recruited through the Amazon Mechanical Turk labor marketplace and were paid for their effort. A total of 312 participated in the study. Sample demographics were $57.0 \%$ male, $42.2 \%$ female, $0.25 \%$ transgender, $0.50 \%$ other; $66 \%$ identified as White, $5 \%$ Hispanic or Latinx, 9.5\% Black, $1 \%$ Native American, and $17.5 \%$ Asian. The sample was generally well educated with $12 \%$ high school, $36 \%$ some college or associate, $40 \%$ bachelor, and $9 \%$ master or doctorate. Finally, the age breakdown was $7 \%$ under $25,54 \% 25-34,26 \% 35-44,8 \%$ $45-54$, and $4 \%$ were 55 and over. Of these participants, seven were eliminated due to incomplete participation, failing attention checks (random responding multiple choice items), or completing the study in an unrealistically short time (-3.0 SDs responding), leaving a final sample of 305 .

\section{RESULTS}

As the choice to hire or not hire the primary diversity candidate was a dichotomous dependent variable, we used logistic regression to test our hypotheses. Clear evidence was obtained for both hypothesized main effects: Higher credential levels $(b=.88, p<.001, \mathrm{OR}=2.41,95 \% \mathrm{CI}$ $[1.52,3.85])$ and the presence of a second diversity candidate for comparison $(b=.58, p=.014, \mathrm{OR}=1.80,95 \%$ CI $[1.13,2.87])$ both corresponded to the primary diversity candidate being selected at a higher rate (see Figure 1). Participants were responding to the stated dual goals of making a good hire and increasing the racial and ethnic diversity of their team. None of the participants chose either applicant $\mathrm{B}$ or $\mathrm{D}$ in any of the scenarios.

The presence of a second diversity candidate increased job offers by $11.1 \%$ and $15.3 \%$ across the weaker and stronger credential conditions, respectively. The manipulations shifted choices across only two candidates the majority candidate with the best credentials and the primary diversity candidate. As this is a between-subjects design, the effects observed are directly attributable to subjects shifting their hiring decision to the diverse candidate. The diversity applicant was more likely to be selected when they had stronger credentials or were evaluated in the presence of a second diversity candidate.

The highest level of endorsement of the primary diversity candidate occurred when a diversity decoy candidate was present $(71.9 \%)$. Although the data suggest a slight interaction, this effect was comparatively small and nonsignificant when we evaluated it in a moderated logistic regression model $(b=.26, \mathrm{OR}=1.29,95 \% \mathrm{CI}[0.51,3.29])$. 


\section{FIGURE 1.}

Percent of diversity job offers by decoy and credential conditions.

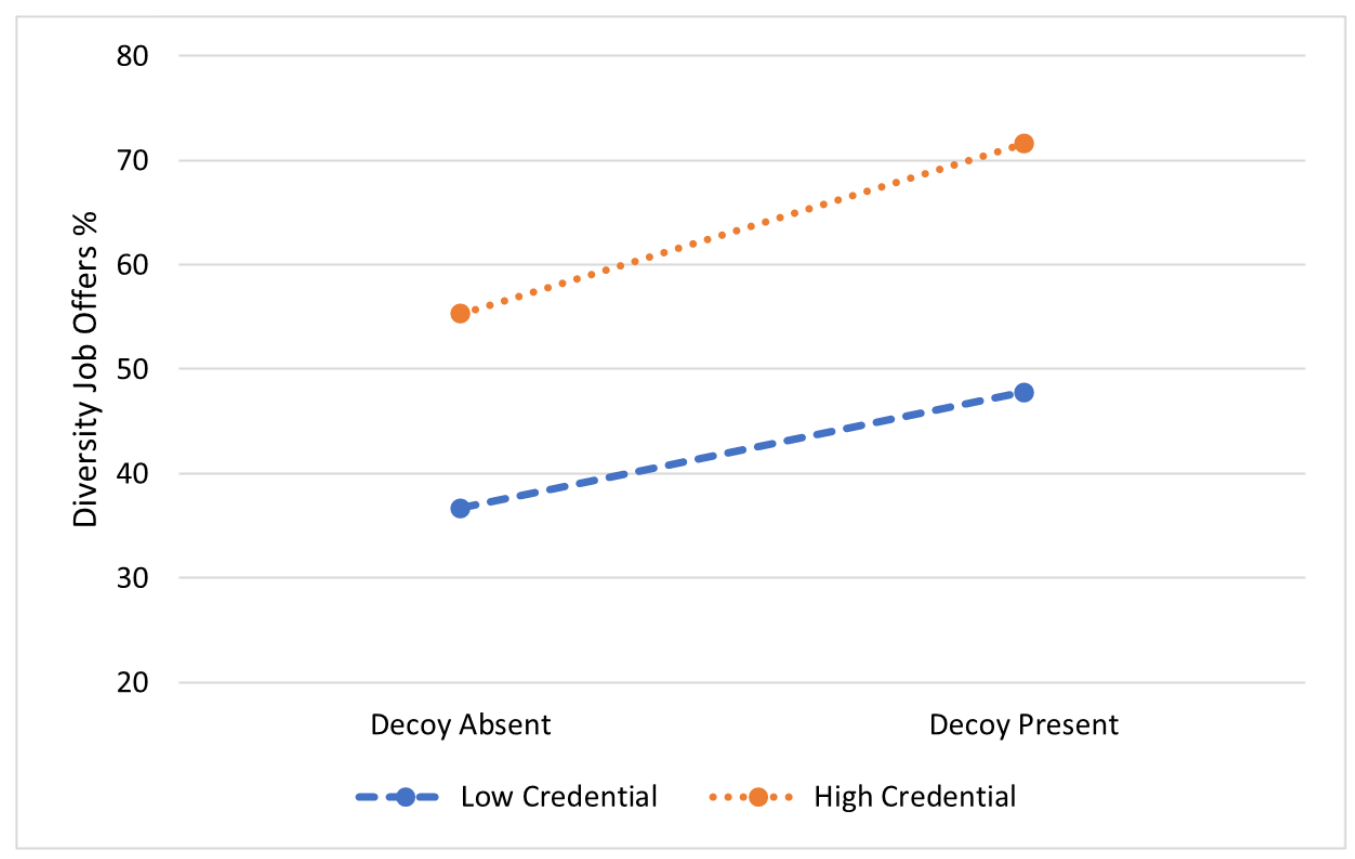

TABLE 2.

Summary of Choice Behaviors by Condition

\begin{tabular}{lccc}
\hline \multirow{2}{*}{ Credential condition } & \multicolumn{2}{c}{ Decoy condition } & \multirow{2}{*}{ Marginal } \\
\cline { 2 - 3 } & Absent & Present & \\
\hline Low-credential diverse candidate & & & \\
$\quad$ Percent who chose diverse candidate & $36.7 \%$ & $47.8 \%$ & $41.9 \%$ \\
$\quad N$ in condition & 79 & 69 & 148 \\
High-credential diverse candidate & & & \\
$\quad$ Percent who chose diverse candidate & $55.3 \%$ & $71.6 \%$ & $63.7 \%$ \\
$\quad N$ in condition & 76 & 81 & 157 \\
Marginal & & & \\
$\quad$ Percent who chose diverse candidate & $45.8 \%$ & $60.7 \%$ & $53.1 \%$ \\
$\quad N$ in condition & 155 & 150 & 305 \\
\hline
\end{tabular}

\section{DISCUSSION}

If decision making were entirely rational and not influenced by comparisons, the presence of the additional diversity candidate in this study should not have had an effect on hiring decisions, as the decoy's credentials were not competitive with any of the primary candidates. Instead, we observed that the primary diversity candidates were chosen at a higher rate when decision makers had another diverse applicant for comparison. The second diversity candidate's presence made the first candidate more desirable or acceptable to decision makers. This effect was observed across credential levels for applicants with relatively weaker or stronger overall credential levels. In the weaker credential 
condition, the two diversity candidate's credentials were both weaker than the best majority candidate, yet offers to the primary diversity candidate increased by $11 \%$ in the presence of the decoy.

To the extent that decision makers view diversity candidates as a separate category of applicants, the presence of a second diverse candidate may help calibrate the decision maker and provide justification. By having a comparison candidate, they can then judge whether or not the first candidate is more or less qualified, within that category. This would be in contrast to viewing all candidates as belonging to the same category but possessing differing levels of compensatory attributes. It is worth noting that selecting the diversity candidate in all cases represented a small loss on at least one attribute; yet, in a two-goal paradigm, that candidate was selected the majority of the time when a second diversity candidate was present. This suggests that a simple loss-aversion explanation is not sufficient.

What is unclear is if individual differences in perceived loss are affecting results. In the high credential case, the trade off in credentials for the diverse candidate is slight. This suggests differences in decision making strategy. The results could be explained by some decision makers operating with a "diversity is a tie breaker" or a similar "select diversity only when there is no loss in credentials" strategy. In contract, some decision makers may use a minimum qualification strategy where they select diverse candidates if the applicant is perceived as minimally qualified. Research on affirmative action indicates that prescriptiveness, which reflects the extent to which race/ethnicity is considered in hiring, moderates the relationship between attitudes toward affirmative action and other variables (Harrison et al., 2006). In this study, the strategy for implementing the goal was left to the decision maker. In light of previous research, it seems likely that prescriptiveness may affect behavior and reactions to the decision making task. It is also worth noting that we focused on the common organizational goal of increasing racial/ethnic diversity. It is also possible that different conceptualizations of diversity may also influence decision making by, again, influencing the relative importance of the two goals.

Theoretically, we are intrigued by an information explanation. The slate of candidates reflects a sampling of the upper tail of the talent distribution. A slate of candidates is a selective draw from a population where the best available are put forward. This gives decision makers information about the nature of the underlying population. It answers the question, "Is this candidate likely to be the best out there?" However, when a single diversity candidate is presented, the decision maker has less information about the distribution of skills in that group. A second diversity candidate may help inform decision making by permitting insight into the distribution of skills within that group. It also provides evidence for justification by having hired the best candidate within the diverse category. The top diverse candidate is presumably the best hire to try to maximize both goals. We think the value of multiple candidates as information for justifying the hire when there are credential tradeoffs is promising.

Although we feel the results are important, our reaction needs to be tempered by a number of potential limitations. The first is the obvious limitation that laboratory results may not generalize to actual hiring decisions. There is some debate as to the generalizability of the general attraction effect (see Frederick et al., 2014; Huber et al., 2014; Simonson, 2014). Some of this criticism is directed at consumer choice examples involving fruits, Kool aid, and jelly beans, and not hiring scenarios where decoy effects have been demonstrated with high fidelity study stimuli (Slaughter et al., 1999). It is worth noting that research has consistently demonstrated that decision-bias research involving undergraduate convenience samples parallels what is found in organizational field samples (Highhouse, 1997). In addition, this study arguably better reflects reality with distinct competing goals and makes salient the importance of external accountability, the latter of which is not present for simple food purchases.

Additionally, other scenarios could be considered within our design to further explore how decoy effects operate. The three candidate strong credential scenario has an element of a decoy effect itself. In the strong credential scenario, $\mathrm{C}$ does dominate $\mathrm{B}$ in skills, knowledge, and diversity. But $\mathrm{C}$ is weaker than $\mathrm{A}$ in knowledge. So we might actually consider B a decoy itself but the additional of the fourth candidate, the focus of our study, still produces a large shift toward the stronger diverse candidate. The choice tension remains because of the skill differential between A and C. A good questions is how do decision makers resolve this?

We see no inherent reason for believing that our sample should yield fundamentally different results compared to samples from other adult populations. Second, although some selection decisions are based on only a couple pieces of information, most consider many pieces of information, especially for college admissions and higher complexity jobs. Although our experiment is closer to real-world scenarios than previous work in this area, the effect observed in the simplified hiring task presented in this experiment may not play out the same in hiring or admissions settings. Whether increased complexity of information may reduce or enhance the effect we observed is unknown. Third, we kept the nature of the diversity characteristic abstract (rather than specifically indicating that "Candidate $\mathrm{C}$ belongs to group X") to reflect the reality that diversity is often an overall goal in many settings. However, it is also possible that the effect may be moderated by the specific diversity goals of the organization or the specific background of the applicant. 
In conclusion, diversity hiring decisions were affected by the credentials of the candidates as well as the presence of a second diversity candidate for consideration. The practical implications of the study are straightforward but important. If organizations are concerned with increasing diversity hiring, they should always consider at least two candidates who would address their diversity goals. Given that the effect occurred even when the diversity candidate had, on average, less desirable credentials, our results suggest that this is a powerful effect.

\section{REFERENCES}

Ariely, D., \& Wallsten, T. S. (1995). Seeking subjective dominance in multidimensional space: An explanation of the asymmetric dominance effect. Organizational Behavior and Human Decision Processes, 63(3), 223-232. https://doi.org/10.1006/ obhd.1995.1075

Bohnet, I., van Geen, A., \& Bazerman, M. (2016). When performance trumps gender bias: Joint vs. separate evaluation. Management Science, 62, 1225-1234.

Faul, F., Erdfelder, E., Buchner, A., \& Lang, A. G. (2009). Statistical power analyses using $G^{*}$ Power 3.1: Tests for correlation and regression analyses. Behavior Research Methods, 41, 11491160.

Frederick, S., Lee, L., \& Baskin, E. (2014). The limits of attraction. Journal of Marketing Research, 51(4), 487-507.

Harrison, D. A., Kravitz, D. A., Mayer, D. M., Leslie, L. M., \& Lev-Arey, D. (2006). Understanding attitudes toward affirmative action programs in employment: Summary and meta-analysis of 35 years of research. Journal of Applied Psychology, 91(5), 1013.

Highhouse, S. (1996). Context-dependent selection: The effects of decoy and phantom job candidates. Organizational Behavior and Human Decision Processes, 65, 68-76.

Highhouse, S. (1997). Understanding and improving job-finalist choice: The relevance of behavioral decision research. Human Resource Management Review, 7, 449-470.

Huber, J., Payne, J. W., \& Puto, C. (1982). Adding asymmetrically dominated alternatives: Violations of regularity and the similarity hypothesis. Journal of Consumer Research, 9, 90-98.

Huber, J., Payne, J. W., \& Puto, C. P. (2014). Let's be honest about the attraction effect. Journal of Marketing Research, 51(4), 520-525.

Huber, J., \& Puto, C. (1983). Market boundaries and product choice: Illustrating attraction and substitution effects. Journal of Consumer Research, 10(1), 31-44.

Kahneman, D., \& Tversky, A. (1979). Prospect theory: An analysis of decision under risk. Econometrica, 47(2), 263-291. https://doi.org/10.2307/1914185

Park, J., \& Kim, J. (2005). The effects of decoys on preference shifts: The role of attractiveness and providing justification. Journal of Consumer Psychology, 15(2), 94-107. https://doi. org/10.1207/s15327663jcp1502_2
Pettibone, J. C., \& Wedell, D. H. (2000). Examining models of nondominated decoy effects across judgment and choice. Organizational Behavior and Human Decision Processes, 81(2), 300-328. https://doi.org/10.1006/obhd.1999.2880.

Rieskamp, J., Busemeyer, J. R., \& Meller, B. A. (2006). Extending the bounds of rationality: Evidence and theories of preferential choice. Journal of Economic Literature, 44, 631-661.

Simonson, I. (1989). Choice based on reasons: The case of attraction and compromise effects. Journal of Consumer Research, 16, 158-174.

Simonson, I. (2014). Vices and virtues of misguided replications: The case of asymmetric dominance. Journal of Marketing Research, 51(4), 514-519.

Slaughter, J. E., Bagger, J., \& Li, A. (2006). Context effects on group-based employee selection decisions. Organizational Behavior and Human Decision Processes, 100, 47-59.

Slaughter, J. E., Sinar, E. F., \& Highhouse, S. (1999). Decoy effects and attribute level inferences. Journal of Applied Psychology, 84, 823-828.

Society for Human Resource Management [SHRM] (2009). Global diversity and inclusion: Perceptions, practices and attitudes. Alexandria, VA: SHRM.

Sunstein, C., \& Thaler, R. (2008). Nudge. New Haven, CT: Yale University Press.

Tversky, A., \& Kahneman, D. (1991). Loss aversion in riskless choice: A reference-dependent model. The Quarterly Journal of Economics, 106, 1039-1061.

Tversky, A., \& Simonson, I. (1993). Context-dependent preferences. Management Science, 39, 1179-1189.

Wedell, D. H., \& Pettibone, J. C. (1996). Using judgments to understand decoy effects in choice. Organizational Behavior and Human Decision Processes, 67, 326-344.

RECEIVED 05/09/19 ACCEPTED 05/29/20 
Appendix A

Study Conditions Varied by Credential Strength and Presence of Additional Diversity

No decoy, weaker credentials

\begin{tabular}{cccc}
\hline Candidate & Organizational skills & Knows the business & Diverse candidate \\
\hline Candidate A & 7 & 8 & No \\
Candidate B & 7 & 6 & No \\
Candidate C & 6 & 5 & Yes \\
\hline
\end{tabular}

No decoy, stronger credentials

\begin{tabular}{cccc}
\hline Candidate & Organizational skills & Knows the business & Diverse candidate \\
\hline Candidate A & 7 & 8 & No \\
Candidate B & 6 & 5 & No \\
Candidate C & 7 & 6 & Yes \\
\hline
\end{tabular}

Diversity decoy, weaker credentials

\begin{tabular}{cccc}
\hline Candidate & Organizational skills & Knows the business & Diverse candidate \\
\hline Candidate A & 7 & 8 & No \\
Candidate B & 7 & 6 & No \\
Candidate C & 6 & 5 & Yes \\
Candidate D & 5 & 5 & Yes \\
\hline
\end{tabular}

Diversity decoy, stronger credentials

\begin{tabular}{cccc}
\hline Candidate & Organizational skills & Knows the business & Diverse candidate \\
\hline Candidate A & 7 & 8 & No \\
Candidate B & 6 & 5 & No \\
Candidate C & 7 & 6 & Yes \\
Candidate D & 5 & 5 & Yes \\
\hline
\end{tabular}

